This document is the unedited Author's version of a Submitted Work that was subsequently accepted for publication in the Journal of the American Chemical Society, copyright (c) American Chemical Society, after peer review. To access the final edited and published work see http://pubs.acs.org/doi/abs/10.1021/jacs.6b10977. 


\title{
Microfluidic Assembly of Monodisperse Vesosomes as Artificial Cell Models
}

\author{
Nan-Nan Deng, Maaruthy Yelleswarapu, Lifei Zheng and Wilhelm T. S. Huck* \\ Radboud University, Institute for Molecules and Materials, Heyendaalseweg 135, 6525 AJ Nijmegen, The Nether- \\ lands
}

Supporting Information

\begin{abstract}
Vesosomes are nested liposomal structures with high potential as advanced drug delivery vehicles, bioreactors and artificial cells. However, to date no method has been reported to prepare monodisperse vesosomes of controlled size. Here we report on a multi-step microfluidic strategy for hierarchically assembling uniform vesosomes from dewetting of double emulsion templates. The exquisite control afforded by our method is illustrated by the formation of concentric, pericentric and multicompartment liposomes. The microfluidic route to vesosomes offers an exceptional platform to build artificial cells as exemplified by the in vitro transcription in "nucleus" liposomes and the mimicry of architecture of eukaryotic cells. Finally, we showed the transport of small molecules across the nucleic envelope via insertion of nanopores into the bilayers.
\end{abstract}

Vesosomes are multicompartmental liposomal structures of varied architectures. ${ }^{1}$ These multivesicular vesicles can consist of multilayered concentric liposomes, or multiple liposomes arranged within or around large liposomes. These structures, as well as related multicompartment polymersomes ${ }^{2}$ and proteinosomes, ${ }^{3}$ have generated significant interest for their potential as drug delivery vehicles ${ }^{1 \mathrm{a}, 4}$, compartmentalized nano- or microscale bioreactors, ${ }^{5}$ or as artificial cell/protocell models. ${ }^{6}$ Zasadzinski and coworkers have shown that vesosomes can protect encapsulated drug carrying compartments from blood components due to the presence of double bilayers, ${ }^{4 \mathrm{~d}}$ and slow vesicle contents release. ${ }^{4 \mathrm{e}}$ Bolinger et al. ${ }^{5 \mathrm{~b}, \mathrm{c}}$ employed multicompartment vesosomes to perform consecutive enzymatic reactions by thermally remote release of different compounds encapsulated in smaller liposomes. Remarkably, vesosomes that contain a "nucleus" or "organelles" represent a promising new concept towards the design of artificial cells, especially the artificial eukaryotic cells. ${ }^{6 a}$ However, conventional methods, ${ }^{7}$ such as bulk hydration ${ }^{8}$ or electroformation ${ }^{9}$ of dried lipid membranes, do not readily yield complex hierarchical vesicular structures, and certainly do not provide any control over dimensions, offer low encapsulation efficiencies and low yields. Moreover, these strategies do not allow a systematic simultaneous loading of different components into diverse compartments. These limitations have severely hindered progress in the application of such multivesicular liposomes. Recently, droplet microfluidics has been shown to offer excellent emulsion templates for the preparation of monodisperse liposomes ${ }^{10}$ as well as polymersomes ${ }^{2 \mathrm{a}, \mathrm{c}, 11}$, but no vesosomes have been reported. 


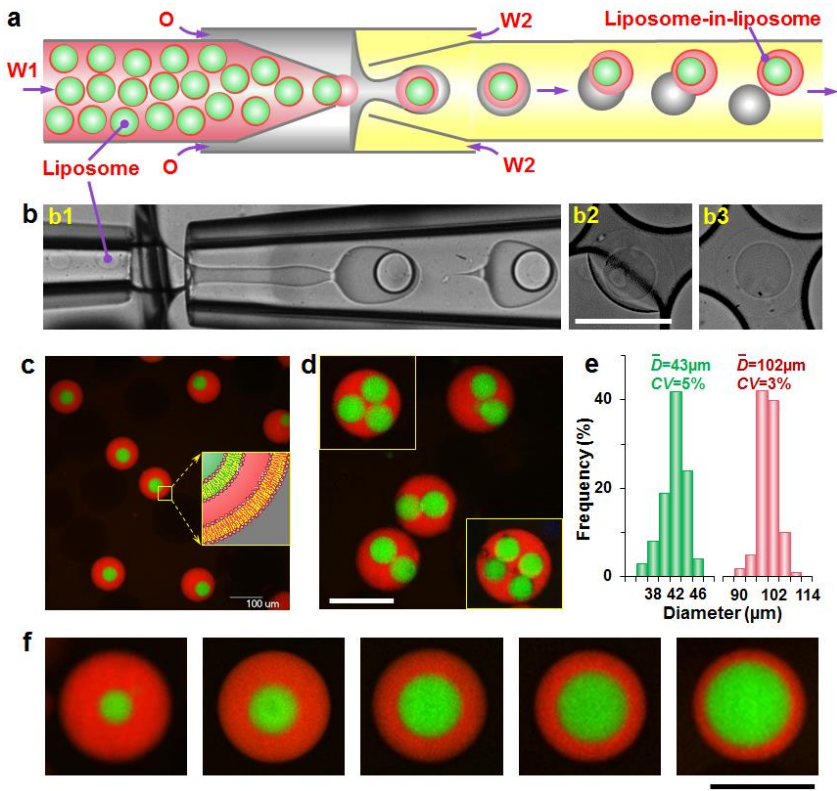

Figure 1. (a) Schematic illustration and (b) snapshots of the microfluidic preparation of double emulsions with an inner liposome and the assembly of vesosomes from emulsion dewetting. (c,d) Confocal images of the monodisperse vesosomes with one (c), two (d), three and four (insets in d) inner liposomes. (e) The size distribution of inner and outer liposomes of the vesosomes in c. (f) As-formed vesosomes with different-sized interior liposomes. Scale bars, $100 \mu \mathrm{m}$.

Recently, we have presented a surfactant-assisted microfluidic strategy for assembling monodisperse liposomes from water-inoil-in-water $(\mathrm{W} / \mathrm{O} / \mathrm{W})$ double emulsion droplets. ${ }^{\text {10a }}$ In this paper, we demonstrate the hierarchical assembly of uniform vesosomes via multi-dewetting processes. Briefly, the single liposomes formed from dewetting of microfluidically prepared W/O/W emulsion droplets were reinjected into the microfluidic devices as the inner phase to fabricate larger W/O/W emulsion droplets loaded with smaller liposomes. The high-order templates will undergo a second dewetting transition to form uniform liposomein-liposome vesicles. The exquisite control afforded by our method is illustrated by the formation of concentric, pericentric and multicompartment liposomes. The resultant vesosomes are potentially used as complex artificial cell models. To this end, coencapsulation of in vitro transcription (IVTx) mix into the "nucleus" liposomes and an in vitro transcription/translation (IVTT) mix into the exterior liposomes was demonstrated. We were able to follow IVTx in real time using RNA aptamers. Finally, we showed the transfer of molecules between vesosome compartments through insertion of membrane proteins into the nucleus bilayers to create nanopores. The microfluidic route to uniform vesosomes of controlled size can facilitate research as diverse as advanced drug delivery systems, artificial cells and microreactors.

To produce monodisperse vesosomes, we first fabricated monodisperse single liposomes as the "nuclei" according to our recent publication. ${ }^{10 a}$ Briefly, we used a glass capillary microfluidic device which comprised of two tapered cylindrical capillaries inserted into opposite ends of a square capillary to prepare W/O/W double emulsion droplets (Figure $\mathrm{S}_{1}$ ) which were utilized to form liposomes (Figure S2) by a dewetting transition. Subsequently, the preformed single liposomes dispersed in an aqueous phase (W1, see SI Materials for details) were reinjected into the microfluidic device as the new inner phase to make larger W/O/W double emulsion droplets (Figures 1a, b1, S2 and Movie S1, see SI Materials for details of the middle oil phase (O) and the outer water phase (W2). In this way, smaller liposomes were successfully loaded within double emulsion droplets. Along with the evaporation of the chloroform fraction of the oil phase, the oil shells of the larger double emulsion droplets gradually dewetted from the interior drops (Figure 1b2), forming vesosomes and separated oil drops with excess lipids (Figure 1 b3). To visualize the vesosomes clearly, fluorescein isothiocyanate-dextran (FITC-Dextran) and rhodamine B isothiocyanate-Dextran (RITC-dextran) were respectively used to label the inner and outer liposomes in some experiments (Figures 1c,d,f). This microfluidic method gives control over the vesosome dimensions and configurations. As Figure 1c shows, the resultant vesosomes are uniform; their internal and external mean diameters are $43 \mu \mathrm{m}$ and $102 \mu \mathrm{m}$ respectively and their coefficients of variation are $5 \%$ and $3 \%$ respectively (Figure 1e). Remarkably, the number of the interior liposomes can be varied by tuning the flow rates. For example, we fixed the flow rates of middle and outer phases at 500 and $4000 \mu \mathrm{L} \mathrm{h}^{-1}$ respectively, and adjusted the inner phase flow rates from 200, 280, 350 to $400 \mu \mathrm{L} \mathrm{h}^{-1}$ to prepare vesosomes containing two, three and four inner liposomes (Figures 1c,d). In addition, vesosomes with diverse dimensions of sub-compartments were also fabricated. As Figures if and S3 show, the shell volumes between interior and exterior liposomes can be easily adjusted, which allow the precise load of ingredients.

Further, the droplet microfluidic technique has shown considerable flexibility in encapsulation of distinct droplets in multiple emulsion droplets, ${ }^{12}$ which offers perfect templates to create structured vesosomes. To demonstrate this idea, we upgraded the microfluidic device with two independent inlets (Figure 2a), ${ }^{10 a}$ which were employed to inject two kinds of liposomes to prepare double emulsion droplets with different inner liposomes. Similarly, these emulsion templates underwent a complete dewetting to generate vesosomes containing distinct liposomes. As Figure $2 \mathrm{~b}$ shows, vesosomes with diverse inner structures (labeled by FITC-Dextran and Alexa Fluor ${ }^{\circledR} 647$ respectively) were successfully achieved. We note that the pairing of reinjected liposomes is the key point to generate vesosomes with controlled numbers and types of inner liposomes. The method can be extended to 
make more complex concentric multicompartment vesosomes, for example, liposome-in-liposome-in-liposome vesosomes (Figures $2 \mathrm{c}$ and $\mathrm{d}$ ).

The vesosomes essentially represent liposomes which contain a "nucleus" or "organelles", and represent a promising new concept towards the design of artificial cells, especially the artificial eukaryotic cells. Although liposomes have been widely employed as protocells or artificial cells to imitate the architectures and functions of prokaryotic cells, such as cell division, ${ }^{13}$ RNA replication ${ }^{14}$ and IVTT, ${ }^{6 \mathrm{~d}}$ the mimicry of intracellular compartmentalization both in structures and functions remains underexplored, mostly because the highly ordered vesicles required, are difficult to make. Furthermore, conventional routes to the vesosomes typically do not efficiently co-encapsulate different solutions into different sub-compartments. Here, we show the advantages of our microfluidic approach in loading diverse complex components and the potential of the resultant vesosomes in building artificial cells.
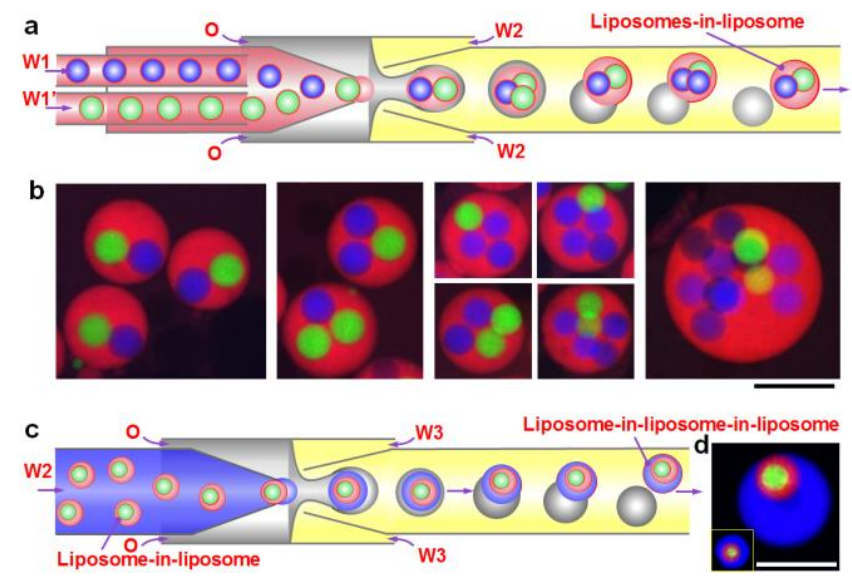

Figure 2. (a) Schematic illustration of the microfluidic preparation of double emulsions with distinct interior liposomes and the dewetting process. (b) Confocal images of the vesosomes with different numbers and ratios of interior liposomes. (c,d) Schematic and confocal images show the formation of triple vesosomes and the resultant structures. Scale bars, $100 \mu \mathrm{m}$.

To mimic the key function of cell nuclei, we encapsulated an IVTx mix in single liposomes to synthesize RNA (Figure za). This mix contains $\mathrm{T}_{7}$ RNAP, pyrophosphatase, DNA template, RNase inhibitor and feeding buffer (see SI IVTx in nucleus liposomes \& IVTT for details), and all components were successfully loaded in inner droplets during the double emulsion template preparation. To visualize the RNA generation, we coded the plasmids with a sequence for Spinach2 aptamer (an RNA mimic of GFP) that can bind a dye called 5-difluoro-4-hydroxybenzylidene imidazolinone (DFHBI) to form a fluorescent complex of Spinach2DFHBI (Figure 3b); both DFHBI and Spinach2 are non-fluorescent until binding occurs. ${ }^{15}$ As Figures 3c, d, S4 and Movie S2 show, the fluorescence intensity of Spinach2-DFHBI in liposomes notably increased from o min to 6o min and up to 120 min during the IVTx. After $2 \mathrm{~h}$ expression, the fluorescence signal reached a plateau probably due to running out of nutrients (Figure $3 \mathrm{~d}$ ). This agrees well with the IVTx reaction in bulk recorded by a plate reader (Figure $\mathrm{S}_{5}$ ).
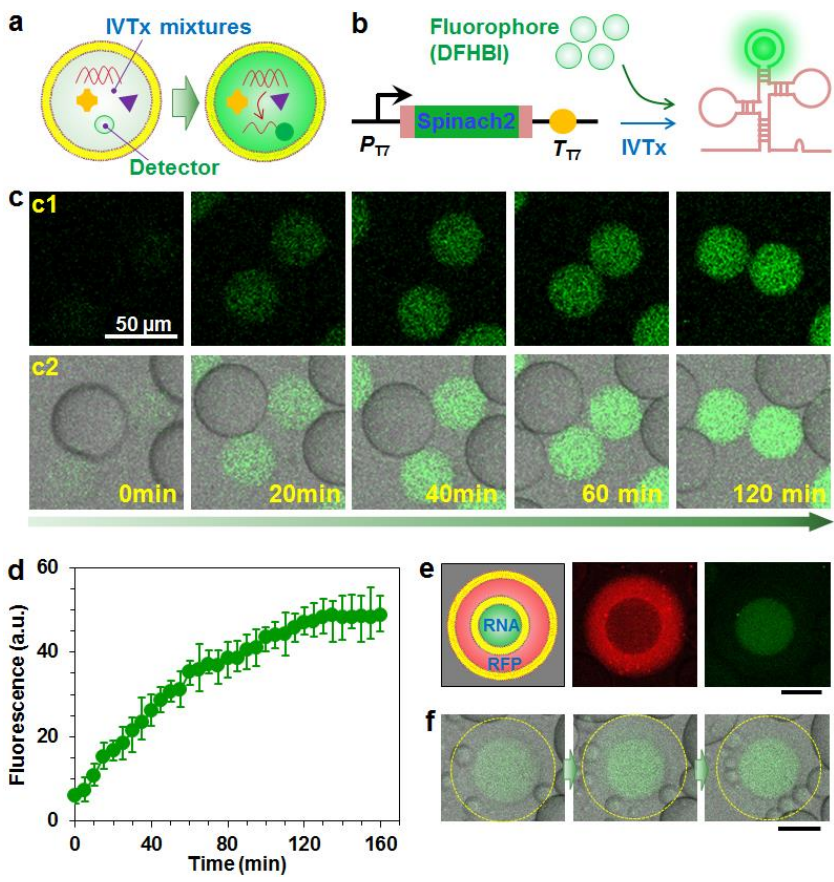
Figure 3. (a,b) Cartoons show in situ detection of IVTx inside the liposomes (a) and the work principle of the RNA aptamer Spinach2. (c) Sequence images of generation of RNA in the liposomes. (d) The RNA expression kinetics. (e) Independent encapsulation of IVTx mix (Spinachz-DFHBI) into interior liposomes and IVTT mix (mRFP) into exterior liposomes. (f) IVTx in nucleus liposome of vesosomes. Scale bars, $50 \mu \mathrm{m}$.
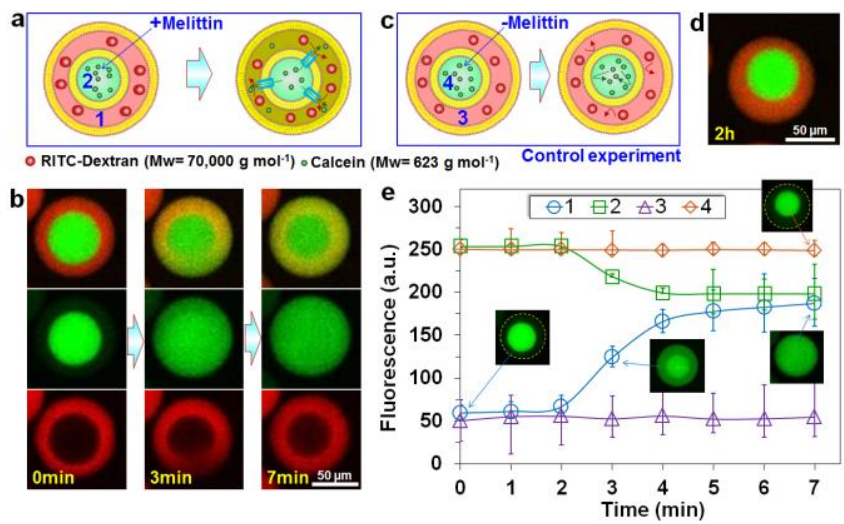

Figure 4. Insertion of membrane pore peptide melittin into the interior bilayers. (a) Cartoons and (b) confocal image series show protein pore-mediated transport of fluorescent molecules from inner liposomes to outer liposomes. (c,d) Control experiment: no melittin was added. (e) Kinetics of time-dependent release of calcein from inner liposome to outer liposome. Curves 1, 2, 3 and 4 mean the fluorescence intensity over time in relevant liposomes as shown in a and c. Scale bars, $50 \mu \mathrm{m}$.

To mimic the architecture of eukaryotic cells, we embedded the single liposomes that contain IVTx mix as artificial nuclei into larger liposomes. In the larger droplets, IVTT mix (consisting of one-third Escherichia coli cell lysate and two-thirds feeding buffer, see SI IVTx in nucleus liposomes \& IVTT for details) ${ }^{16}$ for monomeric red fluorescent protein (mRFP) was encapsulated to form a kind of "cytoplasm" phase (Figure ze). RNA was successfully expressed inside the nucleus liposomes as shown in Figures 3e (last image) and 3f. Moreover, most of the as-formed artificial cell models are stable for more than $8 \mathrm{~h}$, which is sufficient for IVTT or related research. Recently we already demonstrated the in vitro expression of enhanced green fluorescent protein (eGFP) in liposomes. ${ }^{10 a}$ As we here show that transcription and translation can in principle be carried out in two different compartments of the artificial cells, the final key (and probably most challenging) step would be to realize the transfer of RNA across lipid bilayers.

The controlled passage of nutrient molecules and wastes is a key feature of biological cells, in which the nanopores play an important role in both cell envelops and nucleic shells. Therefore, to demonstrate the feasibility of molecule transfer between sub-compartments in vesosomes, we inserted a membrane protein melittin into the nucleus liposomes to create nanopores. Melittin self-assembles into bilayers to create pores of 1-3 nm or 3.5-4.5 nm in diameter depending on the number of assembly subunits, ${ }^{17}$ which allows small molecules to transfer. As Figure 4 a shows, we loaded the inner liposomes with melittin monomers $(2 \mu \mathrm{M})$ and calcein fluorescent molecules $\left(\mathrm{Mw}=623 \mathrm{~g} \mathrm{~mol}^{-1}, 10 \mu \mathrm{M}\right)$ and outer liposomes with RITC-Dextran (average Mw= $70,000 \mathrm{~g} \mathrm{~mol}^{-1}$ ), and then observed the fluorescence intensity over time. Once the nanopores were formed in the nucleus bilayers, the green dye (calcein) in the inner liposomes rapidly (about $2 \mathrm{~min}$ ) diffused into the outer liposomes, while the red dye (RITC-Dextran) in the outer liposome failed to diffuse into the inside because of the larger molecule weight (Figure $4 \mathrm{~b}$, Movie S3). This size exclusion phenomenon also indicates that the morphologies of both the inner and outer liposomes are intact, ensuring that the release of the fluorescent molecules is not because of the burst of inner liposomes. After the dye diffusion, the distribution of calcein in the vesosomes is homogeneous (second row in Figure $4 \mathrm{~b}$ and Figure $4 \mathrm{e}$ ). In contrast, in the absence of membrane proteins, both compartments of the vesosomes only exhibit a slight decay in fluorescence even after $2 \mathrm{~h}$ (Figures $4 \mathrm{c}$-e, Movie S3), may due to photobleaching; no dye transfer was observed. Furthermore, it has been reported that insertion of other membrane proteins into the liposomal bilayers forms nanopores of different sizes, for example, alpha-hemolysin ( $\alpha \mathrm{HL}$ ) creates pores of $1.4 \mathrm{~nm}$ in diameter. ${ }^{18}$ Therefore, it is feasible to selectively insert different membrane proteins, for example, melittin and $\alpha \mathrm{HL}$, into internal and external liposomes respectively to create different nanopores, which will endow different vesosome compartments with different permeability properties. This will open up platforms to study the communications between artificial cell compartments and that between compartment and surrounding environment.

In summary, we have reported a multi-step microfluidic strategy for the hierarchical assembly of uniform vesosomes from dewetting of $\mathrm{W} / \mathrm{O} / \mathrm{W}$ double emulsion droplets. To show the exceptional control over vesosome formation in our method, concentric, pericentric and multicompartment liposomes with distinct contents were successfully created. These structures offer an advanced platform to build artificial cell models as exemplified by the encapsulation of solutions as complex as that for IVTx and IVTT into the different compartments, and real time detection of RNA generation in liposomes. Finally, we showed the insertion of nanopores into the bilayers of the nucleus liposomes, which allows for the transport of small molecules across the nucleic envelopes. Future work will focus on nanopore-mediated transfer of RNA across bilayers and coupled IVTT in vesosome systems. We believe that this approach for fabricating uniform vesosomes with well-defined sizes, will facilitate artificial cell related research like enzyme storage and release, membrane fusion, vesicle transport and multi-step bioprocesses.

\section{ASSOCIATED CONTENT}




\section{Supporting Information}

Supplementary experimental section, figures and movies are available free of charge on the ACS Publications website at DOI:

\section{AUTHOR INFORMATION}

\section{Corresponding Author}

*Wilhelm T.S. Huck (w.huck@science.ru.nl)

Notes

The authors declare no competing financial interests.

\section{ACKNOWLEDGMENT}

This work was supported by the Netherlands Organization for Scientific Research (NWO, TOPPUNT grant 718.014.0o1, the Ministry of Education, Culture and Science (Gravity programme, 024.001.035), and a Marie Skłodowska-Curie Actions Individual Fellowships (H2020-MSCA-IF grant 659907, to N.-N.D.). We thank Sandra Wardle (Radboud University) and Laure Eydieux (Polytech Clermont-Ferrand) for the help with the RNA aptamer.

\section{REFERENCES}

(1) (a)Kisak, E. T.; Coldren, B.; Evans, C. A.; Boyer, C.; Zasadzinski, J. A. Curr. Med. Chem. 2004, 11, 199-219.(b)Torchilin, V.; Weissig, V. Liposomes : a practical approach; Oxford ; New York : Oxford University Press, 2003.

(2) (a)Shum, H. C.; Zhao, Y.-j.; Kim, S.-H.; Weitz, D. A. Angew. Chem., Int. Ed. 2o11, 50, 1648-1651.(b)Chandrawati, R.; Caruso, F. Langmuir 2012, 28, 13798-13807.(c)Kim, S.-H.; Shum, H. C.; Kim, J. W.; Cho, J.-C.; Weitz, D. A. J. Am. Chem. Soc. 2011, 133, 15165-15171.

(3) (a)Liu, X.; Zhou, P.; Huang, Y.; Li, M.; Huang, X.; Mann, S. Angew. Chem. 2o16, 128, 7211-7216.(b)Huang, X.; Patil, A. J.; Li, M.; Mann, S. J. Am. Chem. Soc. 2014, 136, 9225-9234.

(4) (a)Lee, J. S.; Feijen, J. J. Control. Release 2012, 161, 473-483.(b)Marguet, M.; Edembe, L.; Lecommandoux, S. Angew. Chem., Int. Ed. 2012, 51, 1173-1176.(c)Yoo, J.-W.; Irvine, D. J.; Discher, D. E.; Mitragotri, S. Nat. Rev. Drug Discov. 2011, 10, 521-535.(d)Wong, B.; Boyer, C.; Steinbeck, C.; Peters, D.; Schmidt, J.; van Zanten, R.; Chmelka, B.; Zasadzinski, J. A. Adv. Mater. 2o11, 23, 2320-2325.(e)Boyer, C.; Zasadzinski, J. A. ACS Nano 2007, 1, 176-182

(5) (a)Peters, R. J. R. W.; Marguet, M.; Marais, S.; Fraaije, M. W.; van Hest, J. C. M.; Lecommandoux, S. Angew. Chem., Int. Ed. 2o14, 53, 146150.(b)Bolinger, P.-Y.; Stamou, D.; Vogel, H. Angew. Chem., Int. Ed. 20o8, 47, 5544-5549.(c)Bolinger, P.-Y.; Stamou, D.; Vogel, H. J. Am. Chem. Soc. 2004, 126, 8594-8595.(d)Siti, W.; de Hoog, H.-P. M.; Fischer, O.; Shan, W. Y.; Tomczak, N.; Nallani, M.; Liedberg, B. J. Mater. Chem. B 2014, 2, $2733-2737$.

(6) (a)Marguet, M.; Bonduelle, C.; Lecommandoux, S. Chem. Soc. Rev. 2013, 42, 512-529.(b)Choi, H.-J.; Montemagno, C. D. Nano Lett. 2005, 5 , 2538-2542.(c)Elani, Y.; Law, R. V.; Ces, O. Nat. Commun. 2014, 5, 5305.(d)Noireaux, V.; Libchaber, A. Proc. Natl. Acad. Sci. U. S. A. 2004, 101, 17669-17674.(e)Huang, X.; Li, M.; Green, D. C.; Williams, D. S.; Patil, A. J.; Mann, S. Nat. Commun. 2o13, 4, 2239.

(7) (a)Discher, D. E.; Eisenberg, A. Science 20o2, 297, 967-973.(b)Walde, P.; Cosentino, K.; Engel, H.; Stano, P. ChemBioChem 2o10, 11, 848865. (c)Walker, S. A.; Kennedy, M. T.; Zasadzinski, J. A. Nature 1997, 387, 61-64.(d)Paleos, C. M.; Tsiourvas, D.; Sideratou, Z.; Pantos, A. J. Control. Release 2013, 170, 141-152.(e)Kisak, E. T.; Coldren, B.; Zasadzinski, J. A. Langmuir 2002, 18, 284-288.(f)Schneider, M. F.; Marsh, D.; Jahn, W.; Kloesgen, B.; Heimburg, T. Proc. Natl. Acad. Sci. U. S. A. 1999, 96, 14312-14317.(g)Okumura, Y.; Nakaya, T.; Namai, H.; Urita, K. Langmuir 2011, 27, 3279-3282.(h)Paleos, C. M.; Tsiourvas, D.; Sideratou, Z. Langmuir 2012, 28, 2337-2346.

(8) Horger, K. S.; Estes, D. J.; Capone, R.; Mayer, M. J. Am. Chem. Soc. 2009, 131, 1810-1819.

(9) Montes, L. R.; Alonso, A.; Goñi, F. M.; Bagatolli, L. A. Biophys. J. 2007, 93, 3548-3554.

(10) (a)Deng, N.-N.; Yelleswarapu, M.; Huck, W. T. S. J. Am. Chem. Soc. 2016, 138, 7584-7591.(b)Deshpande, S.; Caspi, Y.; Meijering, A. E. C.; Dekker, C. Nat. Commun. 2016, 7, 10447.(c)Shum, H. C.; Lee, D.; Yoon, I.; Kodger, T.; Weitz, D. A. Langmuir 2oo8, 24, 7651-7653.(d)Stachowiak, J. C.; Richmond, D. L.; Li, T. H.; Liu, A. P.; Parekh, S. H.; Fletcher, D. A. Proc. Natl. Acad. Sci. U. S. A. 20o8, 105, 4697-4702.(e)Ota, S.; Yoshizawa, S.; Takeuchi, S. Angew. Chem., Int. Ed. 2oo9, 48, 6533-6537.(f)Abkarian, M.; Loiseau, E.; Massiera, G. Soft Matter 2o11, 7, 4610-4614.(g)Hu, P. C.; Li, S.; Malmstadt, N. ACS Appl. Mat. Interfaces 2011, 3, 1434-144o.(h)Funakoshi, K.; Suzuki, H.; Takeuchi, S. J. Am. Chem. Soc. 2007, 129, 126o812609.(i)Tan, Y.-C.; Hettiarachchi, K.; Siu, M.; Pan, Y.-R.; Lee, A. P. J. Am. Chem. Soc. 2006, 128, 5656-5658.

(11) (a)Shum, H. C.; Kim, J.-W.; Weitz, D. A. J. Am. Chem. Soc. 20o8, 130, 9543-9549.(b)Thiele, J.; Chokkalingam, V.; Ma, S.; Wilson, D. A.; Huck, W. T. S. Mater. Horiz. 2014, 1, 96-101.

(12) (a)Deng, N.-N.; Wang, W.; Ju, X.-J.; Xie, R.; Weitz, D. A.; Chu, L.-Y. Lab Chip 2013, 13, 4047-4052.(b)Wang, W.; Xie, R.; Ju, X.-J.; Luo, T.; Liu, L.; Weitz, D. A.; Chu, L.-Y. Lab Chip 2011, 11, 1587-1592.

(13) (a)Osawa, M.; Anderson, D. E.; Erickson, H. P. Science 20o8, 320, 792-794.(b)Kurihara, K.; Tamura, M.; Shohda, K.-i.; Toyota, T.; Suzuki, K.; Sugawara, T. Nat. Chem. 2011, 3, 775-781.

(14) Chen, I. A.; Salehi-Ashtiani, K.; Szostak, J. W. J. Am. Chem. Soc. 2005, 127, 13213-13219.

(15) Strack, R. L.; Disney, M. D.; Jaffrey, S. R. Nat Meth 2013, 10, 1219-1224.

(16) Sokolova, E.; Spruijt, E.; Hansen, M. M. K.; Dubuc, E.; Groen, J.; Chokkalingam, V.; Piruska, A.; Heus, H. A.; Huck, W. T. S. Proc. Natl. Acad. Sci. U. S. A. 2013, 110, 11692-11697.

(17) (a)Ladokhin, A. S.; Selsted, M. E.; White, S. H. Biophys. J. 1997, 72, 1762-1766.(b)Matsuzaki, K.; Yoneyama, S.; Miyajima, K. Biophys. J. 1997, 73, 831-838.

(18) Song, L.; Hobaugh, M. R.; Shustak, C.; Cheley, S.; Bayley, H.; Gouaux, J. E. Science 1996, 274, 1859-1865. 